\title{
Atitudes de enfermeiros de cuidados primários frente à doença mental: comparação Brasill-Portugal
} Attitudes of Primary Health Care nurses towards mental illness: Brazil-Portugal comparison Actitudes de enfermeros de atención primaria de salud con relación a personas con enfermedad mental: comparación Brasil-Portugal

Maria Do Perpétuo Socorro de Sousa Nóbrega1 in https://orcid.org/0000-0002-4974-0611 Carla Sílvia Neves da Nova Fernandes ${ }^{2}$ io https://orcid.org/0000-0001-7251-5829

Estela Duarte ${ }^{1}$ io https://orcid.org/0000-0003--3859-4599

Wanderson Carneiro Moreira ${ }^{1}$ io https://orcid.org/0000-0003-2474-1949 Suellen Cristina da Silva Chaves ${ }^{1}$ io hitps://orcid.org/0000-0003--3234-9752

\section{Como citar:}

Nóbrega MP, Fernandes CS, Duarte E, Moreira WC, Chaves SC. Atitudes de enfermeiros de cuidados primários frente à doença mental: comparação Brasil-Portugal. Acta Paul Enferm. 2020;33:eAPE20190145.

DOI

http://dx.doi.org/10.37689/actaape/2020A001456

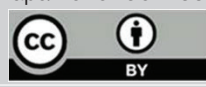

Descritores Atitude do pessoal de saúde; Atenção primária à saúde; Saúde mental; Enfermagem Psiquiátrica; Transtornos mentais; Brasil; Portugal

Keywords

Attitude of health personnel; Primary health care; Mental health; Psychiatric nursing; Mental disorders; Brazil; Portugual

Descriptores

Actitud del personal de salud; Atención primaria de salud; Salud mental; Enfermería psiquiátrica; Trastornos mentales; Brasil; Portugual

Submetido

13 de Junho de 2019

Aceito

16 de Março de 2020

Autor correspondente

Maria do Perpétuo S. S. Nóbrega

E-mail: perpetua.nobrega@usp.br

\section{Resumo}

Objetivo: Analisar comparativamente as atitudes e as experiências de enfermeiros de cuidados primários de Brasil e Portugal frente à pessoa com doença mental.

Métodos: Estudo transversal, quantitativo realizado com 500 enfermeiros de cuidados primários. A coleta dos dados ocorreu por meio de questionário sócio demográfico e da escala "Opiniões sobre a Doença Mental". Aplicou-se estatística descritiva e correlacional, com os testes de Mann-Whitney e correlação de Spearman $(p<0,05)$.

Resultados: Os enfermeiros brasileiros e portugueses demonstraram atitudes positivas ao apresentar, no total das dimensões da escala, pontuação abaixo da média $(43 \%)$ e (74\%), respectivamente, exceto nas dimensões Autoritarismo ( $M=44,6)$, Restrição Social ( $M=42,0$ e e de Etiologia Interpessoal $(M=30,6)$ frente à pessoa com doença mental, os enfermeiros brasileiros apresentaram média mais elevada.

Conclusão: No geral, as atitudes em ambos os países em relação às pessoas com doença mental são positivas, exceto na dimensão Autoritarismo. As experiências de cuidados interferem nas atitudes dos enfermeiros nos dois países. Atitudes negativas devem ser reconhecidas e desconstruídas para inclusão da saúde mental nos cuidados primários.

\section{Abstract}

Objective: To analyze comparatively the attitudes and experiences of Primary Health Care nurses from Brazil and Portugal towards people with mental illness.

Methods: This cross-sectional and quantitative study was conducted with 500 Primary Health Care nurses. Data were collected through a socio-demographic questionnaire and the Opinions about Mental Illness scale. Descriptive and correlational statistics were applied using Mann-Whitney and Spearman correlation tests $(p<0.05)$.

Results: Brazilian and Portuguese nurses showed positive attitudes by presenting, in the total dimensions of the scale, a score below the mean, $43 \%$ and $74 \%$, respectively, except in the Authoritarianism ( $M=44.6)$, Social Restriction ( $M=42.0)$ and Interpersonal Etiology ( $M=30.6)$ dimensions. Compared to people with mental illness, Brazilian nurses had a higher mean.

Conclusion: Overall, attitudes in both countries towards people with mental illness are positive, except in the Authoritarianism dimension. Care experiences interfere with the attitudes of nurses in both countries. Negative attitudes should be recognized and deconstructed for the inclusion of mental health in Primary Health Care. 


\section{Resumen}

Objetivo: Analizar comparativamente las actitudes y experiencias de enfermeros de atención primaria de Brasil y Portugal con relación a personas con enfermedad mental.

Métodos: Estudio transversal, cuantitativo realizado con 500 enfermeros de atención primaria. La recolección de datos ocurrió mediante un cuestionario sociodemográfico y con la escala "Opiniones sobre enfermedades mentales". Se aplicó estadística descriptiva y correlacional, con las pruebas de MannWhitney y correlación de Spearman $(p<0,05)$.

Resultados: Los enfermeros brasileños y portugueses demostraron actitudes positivas al presentar una puntuación por debajo del promedio en el total de las dimensiones de la escala (43 \% y $74 \%$, respectivamente), excepto en las dimensiones Autoritarismo $(M=44,6)$, Restricción social ( $M=42,0)$ y Etiología interpersonal $(M=30,6)$ con relación a personas con enfermedad mental, que los enfermeros brasileños presentaron un promedio más elevado.

Conclusión: En general, las actitudes de ambos países con relación a las personas con enfermedad mental son positivas, excepto en la dimensión Autoritarismo. Las experiencias de la atención interfieren en las actitudes de los enfermeros en los dos países. Las actitudes negativas deben reconocerse y deconstruirse para que haya una inclusión de la salud mental en la atención primaria.

\section{Introdução}

No mundo, aproximadamente 650 milhóes de pessoas preenchem critérios diagnósticos para doença mental, ${ }^{(1)}$ o que representa um importante problema de saúde pública devido sua cronicidade e dificuldades no tratamento. ${ }^{(2,3)}$ As doenças mentais caracterizam-se por mudanças comportamentais aparentes que podem gerar atitudes negativas e crenças associadas. $^{(4)}$

A crença de que pessoas com doenças mentais são perigosas e responsáveis por sua própria doença, desencadeia estereótipos em relação a essa condição clínica. ${ }^{(3)}$ Ao concordar com determinado estereótipo e assumir emocionalmente essa ideia, em decorrência de alguns comportamentos, como o medo de pessoas com doença mental, estabelece-se o preconceito. ${ }^{(5)}$

$\mathrm{O}$ estigma ligado à doença mental compromete o bem-estar biopsicossocial e emocional da pessoa, da família e do cuidador, bem como seu comportamento na busca de cuidados de saúde. ${ }^{(4)}$ No campo da saúde mental, observa-se o estigma, tanto da população em geral, como dos profissionais de saúde, e estes externam atitudes mais negativas em relação ao progresso do tratamento, ${ }^{(6,7)}$ dificultando sua evolução. ${ }^{(8)}$

A conduta estigmatizante tem por base um componente sociocultural frente a algo e envolve domínios afetivos, cognitivos e comportamentais diante de uma situação social, é considerada uma resposta a um estímulo que pode influenciar negativamente a integração social do indivíduo, ou positivamente, quando fornece apoio à pessoa com doença mental. ${ }^{(9)}$ Considerando que os enfermeiros de serviços primários de saúde estão na linha de frente da assis- tência e que suas atitudes podem interferir na qualidade dos cuidados que promovem, ${ }^{(7)}$ é fundamental que estejam preparados e apoiados para constituir atitudes positivas, refletidas em relaçóes terapêuticas que constituam indicador de resultados para a melhoria do tratamento/reabilitação. ${ }^{(4)}$

Estudos que investigam e contribuem para promover mudança de atitudes relacionadas a problemas de saúde estigmatizados são necessários para estimular práticas que fortaleçam o cuidado, pautadas por meio de formação continuada e de capacitaçóes direcionadas, ${ }^{(10)}$ corroborando desse modo, a relevância de compreender como o fenômeno atitudes se processa. ${ }^{(6)}$ Em revisão de literatura foram encontrados estudos sobre atitudes de profissionais de cuidados primários, porém, alguns não contemplam a opiniâo de enfermeiros: na China estudo com provedores de cuidado de saúde primários aponta atitudes pessimistas e negativas para com pessoas com doença mental, ${ }^{(6)}$ na Finlândia, ${ }^{(10)}$ e África do Sul, ${ }^{(11)}$ enfermeiros de cuidados primários têm atitudes positivas em relação a essa clientela. Salienta-se que nenhum desses estudos utilizou a escala Opinióes sobre a Doença Mental (ODM), aqui proposto.

Estudo comparativo entre países europeus, ${ }^{(12)}$ incluindo Portugal, faz uma aproximaçáo com a temática atitude, porém não evidencia especificamente a realidade das atitudes de enfermeiros de cuidados primários. No Brasil, inexistem estudos com enfermeiros de cuidados primários frente à doença mental, e os que se aproximam foram realizados com enfermeiros de unidades psiquiátricas e de urgência/emergência geral, ${ }^{(13,14)}$ com uso da escala ODM. Assim, pouco se sabe nos dois países sobre as 
atitudes que os enfermeiros de cuidados primários têm sobre as pessoas com doenças mentais e suas experiências de cuidados, constituindo lacuna de investigação que determine a essência das atitudes desse grupo.

Na conjuntura das reformas políticas de saúde mental, Brasil e Portugal, se aproximam quanto à pauta inserção da saúde mental na atenção primária, ${ }^{(15)}$ mas para sustentá-la devem aproximar os enfermeiros de cuidados primários das necessidades de pessoas com doenças mentais e investir em conhecimentos/competências que os auxiliem a identificar, cuidar e encaminhar pessoas com doenças mentais aos serviços especializados de forma qualificada. Portanto, objetiva-se com esse estudo analisar comparativamente as atitudes e as experiências de enfermeiros de cuidados primários de Brasil e Portugal frente à pessoa com doença mental.

\section{Métodos}

Estudo transversal, descritivo, quantitativo, multicêntrico, desenvolvido em seis Unidades de Saúde Familiar da Administração Regional de Saúde do Norte, Porto/Portugal, e de 69 Unidades Básicas de Saúde das seis Coordenadorias Regionais de Saúde de São Paulo, São Paulo/Brasil.

Os participantes do estudo foram enfermeiros recrutados por meio de amostra não probabilística. Os critérios de inclusão foram: ser enfermeiro, independentemente do tempo de atuação profissional e no serviço, atuando na gestão e/ou assistência. Um total de 500 enfermeiros apresentaram critérios de elegibilidade nos dois países, sendo 250 de cada.

A coleta de dados foi realizada de abril a agosto de 2018, nos dois países, simultaneamente, por meio de formulário do Googledocs, contendo dados sócio demográficos: sexo, idade, estado civil, habilitações acadêmicas, tempo de formação, de atuação no atual serviço, carga de trabalho semanal, experiência/frequência com que se depara com pessoas com doenças mentais. Inicialmente, foi realizado contato telefônico com os coordenadores dos serviços de saúde para falar sobre a pesquisa, que divulgasse aos enfermeiros e solicitasse participação voluntária na mesma, com devolutiva positiva. Para garantir o sigilo na coleta, foi enviado aos coordenadores dos serviços o link para o Googledocs (formulários de pesquisa, roteiro orientador para preenchimento e termo de consentimento livre e esclarecido) para que repassasse aos correios eletrônicos dos enfermeiros.

As atitudes frente à doença mental foram levantadas pela escala "Opinions about Mental Illness". (16) A ODM foi desenvolvida por Struening e Cohen em 1963, sendo bastante utilizada na literatura nacional e internacional. Para uso no Brasil, a ODM foi traduzida e validada para o português por Rodrigues (1983), ${ }^{(17)}$ sob o título "Escala de Opinióes sobre a Doença Mental (ODM)”. Por ser de domínio público no Brasil e em Portugal, não houve necessidade de solicitar autorização aos autores.

A ODM é composta por 7 dimensões: Autoritarismo com 11 perguntas a cerca da opinião sobre o sujeito com doença mental pertencer a uma "classe de pessoas com valor inferior"; Benevolência com 14 perguntas sobre paternalismo em relação ao indivíduo com doença mental; Ideologia da Higiene Mental com 9 perguntas sobre a percepçáo do doente mental como sendo uma "pessoa normal"; Restrição Social 7 perguntas sobre doente mental x periculosidade para a sociedade; e Etiologia Interpessoal com 10 perguntas frente a crença de que a doença mental é resultante das más experiências interpessoais vividas na infância; No total são 51 perguntas. Apresenta amplitude de 51 a 306, com ponto médio 178,5. Quanto maior for a média (ponto médio) obtida, mais negativas são as atitudes, à exceção das dimensôes Benevolência e Ideologia da Higiene Mental.

Os dados foram codificados, limpos e analisados com o Software for Windows Statistical Package for the Social Sciences (SPSS) versão 25. Aplicou-se estatística descritiva e correlacional para verificar a correlação entre as variáveis sócio demográficas/laborais e as dimensóes da ODM nas amostras. Aplicou-se o teste de Mann-Whitney e correlação de Spearman. Adotou-se o nível de confiança de $95 \%$ o qual apresentou significância entre os resultados com valor de $\mathrm{p}<0,05$.

O estudo obedeceu a todos os princípios éticos em pesquisa sendo aprovado pelo Comitê de Ética 
em Pesquisa com Seres Humanos da Faculdade Fernando Pessoa de Porto/Portugal (parecer no 155 2017), e da Escola de Enfermagem da Universidade de São Paulo (Parecer 2.384.303), em conformidade com a Declaraçáo de Helsinque. Todos os participantes assinaram termo de consentimento para participação.

\section{Resultados}

A partir dos achados observam-se importantes diferenças entre Brasil e Portugal especialmente quanto ao fato dos enfermeiros portugueses serem mais velhos, terem mais tempo de formado, possuírem estabilidade no emprego, e muitos possuírem o título de mestrado. As variáveis sócio demográficas em comum entre as duas realidades são: sexo feminino, casadas, idade variando entre 36 e 42 anos, e que já tiveram experiência com pessoas com doença mental.

Quanto ao tempo de formação e de atuação no serviço atual, os enfermeiros portugueses apresentaram maior frequência $(10-20$ anos $=34,8 \%)$ e $(10$ 20 anos $=36,4 \%)$, do que brasileiros (5-10 anos= $39,2 \%)$ e $(1-5$ anos $=42,8 \%)$. Em relaçáo à carga horária semanal de trabalho, os enfermeiros brasileiros tiveram média superior $(40 \mathrm{hs}=62,8 \%)$ do que os portugueses $(35 \mathrm{hs}=54,4 \%)$. Assim como a frequência com que se deparam com pessoas com doenças mentais (diariamente $=63,3 \%$ ) e Portugal (semanalmente $=34,3 \%)$. Em relação às habilitaçóes acadêmicas, os enfermeiros portugueses têm maior número de mestres $(18,0 \%)$ (Tabela 1$)$.

Os enfermeiros portugueses têm mais experiência com pessoas com doenças mentais $(94,8 \%)$, se deparam semanalmente com pessoas com esta condição $(34,8 \%)$, consideram adequado seus conhecimentos sobre as necessidades de cuidados de saúde com essa clientela $(53,2 \%)$, julgam inadequada a avaliaçáo das necessidades de saúde e a assistência prestada nos serviços onde atuam $(63,6 \%)$ e $(54,8 \%)$, respectivamente.

Os enfermeiros brasileiros acreditam que seu conhecimento sobre as necessidades de cuidados de saúde é muito adequado (42,0\%), consideram que os cuidados de saúde primários prestados em
Tabela 1. Caracterização sociodemográfica da amostra, estudo Atitudes APS, Porto-Portugal e São Paulo-Brasil $(n=500)$ enfermeiros

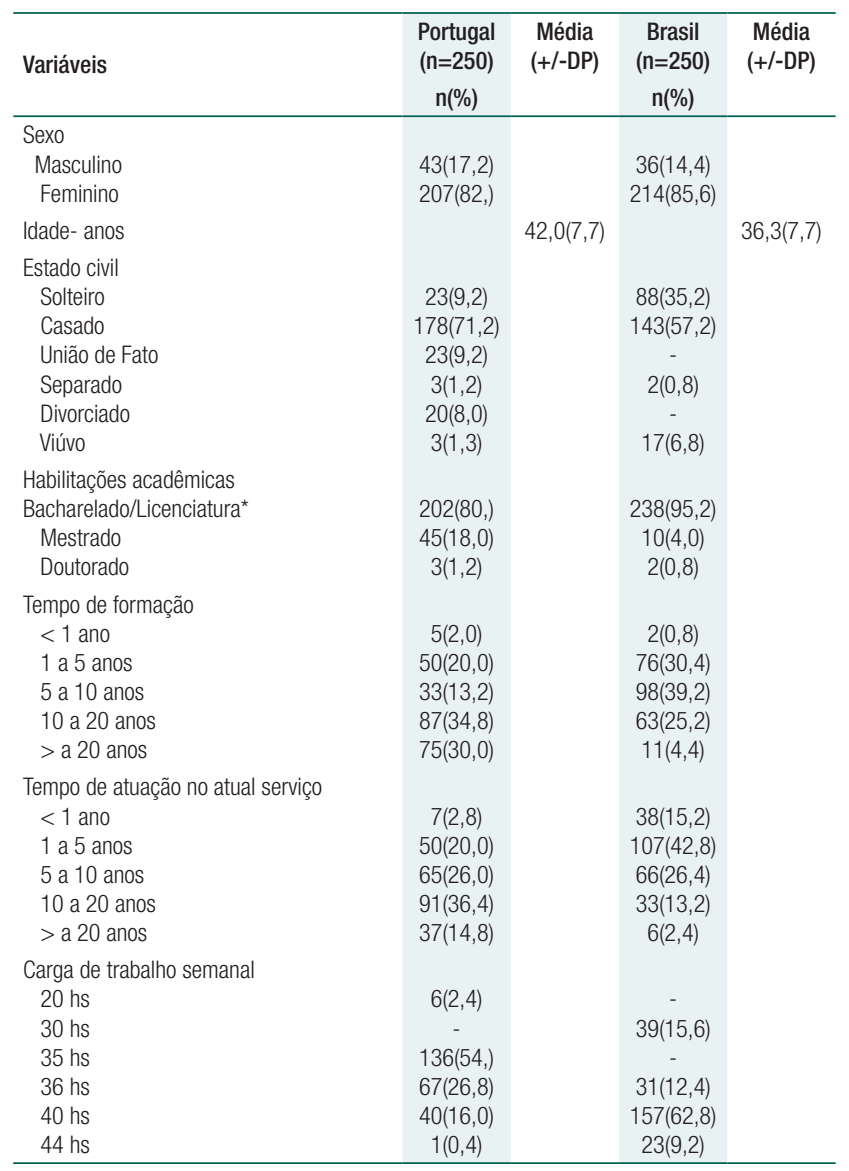

*Licenciatura em Portugal equivale ao Bacharelado no Brasil

saúde mental são muito adequados $(50,4 \%)$, dados superiores ao universo português. Também julgam que a avaliação sobre as necessidades de saúde das pessoas com doenças mentais é conduzida de forma adequada $(33,6 \%)$, a assistência disponibilizada pelos seus serviços é adequada $(40,4 \%)$ e muito adequada $(31,6 \%)$, demonstrando contrastes relevantes (Tabela 2).

\section{Opinião sobre a doença mental}

Os enfermeiros brasileiros apresentam ponto mínimo da ODM de 124 e máximo 260, com média de 197, acima do valor médio da escala que é de 178,5 e desvio padrão de 17,2, o que permite estabelecer que no global, cerca de $43 \%$ dos enfermeiros brasileiros apresentam médias positivas, ou seja, tem atitudes positivas em relação à doença mental. Os enfermeiros portugueses apresentaram ponto mínimo da ODM de 106 e ponto máximo de 220, com 
Tabela 2. Caracterização das experiências de enfermeiros de cuidados primários frente a pessoa com doença mental, estudo Atitudes APS, Porto-Portugal e São Paulo-Brasil $(n=500)$ enfermeiros

\begin{tabular}{|c|c|c|}
\hline Variáveis & $\begin{array}{c}\begin{array}{c}\text { Portugal } \\
(n=250)\end{array} \\
n(\%)\end{array}$ & $\begin{array}{c}\begin{array}{c}\text { Brasil } \\
(n=250)\end{array} \\
n(\%)\end{array}$ \\
\hline \multicolumn{3}{|c|}{ Experiência com pessoas com doenças mentais } \\
\hline $\begin{array}{l}\text { Não } \\
\text { Sim }\end{array}$ & $\begin{array}{c}13(5,2) \\
237(94,8)\end{array}$ & $\begin{array}{r}91(36,4) \\
159(63,6)\end{array}$ \\
\hline \multicolumn{3}{|c|}{$\begin{array}{l}\text { Frequência que se depara com pessoas com doenças } \\
\text { mentais }\end{array}$} \\
\hline $\begin{array}{l}\text { Diariamente } \\
\text { Semanalmente } \\
\text { Mensalmente } \\
\text { Ocasionalmente }\end{array}$ & $\begin{array}{l}65(27,2) \\
82(34,3) \\
67(28,0) \\
25(10,5)\end{array}$ & $\begin{array}{c}159(63,6) \\
76(30,4) \\
15(6,0) \\
-\end{array}$ \\
\hline \multicolumn{3}{|c|}{$\begin{array}{l}\text { Conhecimento sobre necessidades de cuidados de saúde } \\
\text { à pessoa com doença mental }\end{array}$} \\
\hline $\begin{array}{l}\text { Muito inadequado } \\
\text { Inadequado } \\
\text { Adequado } \\
\text { Muito adequado }\end{array}$ & $\begin{array}{c}10(4,0) \\
104(41,6) \\
133(53,2) \\
3(1,2)\end{array}$ & $\begin{array}{c}33(13,2) \\
14(5,6) \\
98(39,2) \\
105(42,0)\end{array}$ \\
\hline \multicolumn{3}{|c|}{$\begin{array}{l}\text { Conhecimento sobre o papel dos cuidados de saúde } \\
\text { primária na assistência em saúde mental }\end{array}$} \\
\hline $\begin{array}{l}\text { Muito inadequado } \\
\text { Inadequado } \\
\text { Adequado } \\
\text { Muito adequado }\end{array}$ & $\begin{array}{c}10(4,0) \\
113(45,2) \\
119(47,6) \\
8(3,2)\end{array}$ & $\begin{array}{c}22(8,8) \\
15(6,0) \\
87(34,8) \\
126(50,4)\end{array}$ \\
\hline \multicolumn{3}{|c|}{$\begin{array}{l}\text { Avaliação das necessidades de saúde das pessoas com } \\
\text { doenças mentais }\end{array}$} \\
\hline $\begin{array}{l}\text { Muito inadequado } \\
\text { Inadequado } \\
\text { Adequado } \\
\text { Muito adequado }\end{array}$ & $\begin{array}{c}23(9,2) \\
159(63,6) \\
8(3,2) \\
60(24,0)\end{array}$ & $\begin{array}{l}60(24,0) \\
37(14,8) \\
84(33,6) \\
69(27,6)\end{array}$ \\
\hline \multicolumn{3}{|c|}{$\begin{array}{l}\text { Avaliação da assistência a pessoas com doenças mentais } \\
\text { disponibilizadas pelos seus serviços }\end{array}$} \\
\hline $\begin{array}{l}\text { Muito inadequado } \\
\text { Inadequado } \\
\text { Adequado } \\
\text { Muito adequado }\end{array}$ & $\begin{array}{c}28(11,2) \\
137(54,8) \\
5(2,0) \\
80(32,0)\end{array}$ & $\begin{array}{c}33(13,2) \\
37(14,8) \\
101(40,4) \\
79(31,6)\end{array}$ \\
\hline
\end{tabular}

média de 117,0 , abaixo do valor médio da escala que é de 178,5 e desvio padrão de 13,8 , o que permite estabelecer que cerca de $74 \%$ dos enfermeiros portugueses também apresentam médias positivas, ou seja, atitudes positivas no geral frente à doença mental (Tabela 3). Em relação às dimensōes da escala ODM, os enfermeiros portugueses apresentam atitudes ligeiramente mais Benevolentes $(\mathrm{M}=64,41)$ e de Ideologia da Higiene Mental $(M=39,35)$ do que brasileiros. Já os enfermeiros brasileiros têm mais atitudes de Autoritarismo $(\mathrm{M}=44,55)$, de Restrição Social $(M=42,04)$ e de Etiologia Interpessoal $(\mathrm{M}=30,62)$ frente à pessoa com doença mental, comparada aos portugueses.

\section{Opiniões sobre a doença mental $x$ variáveis específicas}

$\mathrm{Na}$ comparação entre os dois países, a carga horária de trabalho semanal associa-se as atitudes negativas para os enfermeiros brasileiros, sendo $(\mathrm{p}=0,324)$ de $40 \mathrm{~h} / \mathrm{sem}(62,8 \%)$ em relação aos portugueses. O tempo de formação $(\mathrm{p}=0,198)$, de 5 a 10 anos $(39,2 \%)$, se relaciona de modo positivo, expressando-se em atitudes mais negativas. A frequência com que os enfermeiros brasileiros se deparam com pessoas com doenças mentais $(\mathrm{p}=0,227)$ se correlaciona de modo positivo com o menor tempo de atuação no serviço atual $(42,8 \%)$, expressando-se em atitudes mais negativas.

A avaliação das necessidades de saúde conduzida no serviço $(p=-0,157)$ se relaciona de modo negativo com a visão de que essa avaliação é conduzida adequadamente $(33,6 \%)$, expressando-se em atitudes mais negativas frente à pessoa com doença mental, contrariando os níveis elevados nas dimensôes Autoritarismo e Restrição Social. Quanto ao gênero, em Portugal, homens pontuam em média 2,68 a

Tabela 3. Total e Dimensões da ODM, estudo Atitudes APS, Porto-Portugal e São Paulo-Brasil $(n=500)$ enfermeiros

\begin{tabular}{|c|c|c|c|c|c|c|c|c|c|c|c|c|c|c|c|c|}
\hline \multirow[b]{2}{*}{ Dimensão } & \multicolumn{8}{|c|}{ Portugal $(n=250)$} & \multicolumn{8}{|c|}{ Brasil $(n=250)$} \\
\hline & $x$ & DP & Min & Q1 & Med & Q3 & Máx & $\underset{(95 \%)}{\text { IC }}$ & $\mathrm{x}$ & DP & Min & Q1 & Med & Q3 & Máx & $\begin{array}{c}\text { IC } \\
(95 \%)\end{array}$ \\
\hline Escala total & 177,04 & 13,83 & 138 & 168,2 & 177 & 185 & 222 & $\begin{array}{c}175- \\
178,5\end{array}$ & 197,02 & 17,25 & 121 & 188 & 198 & 208 & 260 & $\begin{array}{c}195,5- \\
200\end{array}$ \\
\hline Autoritarismo & 31,04 & 6,56 & 17 & 26 & 31 & 36 & 50 & $\begin{array}{c}30,22- \\
31,86\end{array}$ & 44,55 & 6,23 & 23 & 41 & 45 & 49 & 63 & $\begin{array}{c}43,78- \\
45,3\end{array}$ \\
\hline Benevolência & 64,41 & 6,97 & 26 & 61 & 65 & 69 & 78 & $\begin{array}{c}64- \\
65,5\end{array}$ & 51,68 & 5,37 & 31 & 48 & 52 & 55 & 68 & $\begin{array}{c}51- \\
52,5\end{array}$ \\
\hline Ideologia de higiene mental & 39,35 & 4,71 & 23 & 37 & 39 & 43 & 51 & $\begin{array}{c}38,76- \\
39,93\end{array}$ & 28,12 & 3,97 & 18 & 25 & 28 & 31 & 42 & $\begin{array}{c}27,63- \\
28,6\end{array}$ \\
\hline Restrição social & 24,90 & 6,05 & 10 & 21 & 25 & 29 & 44 & $\begin{array}{c}24,14- \\
25,65\end{array}$ & 42,04 & 5,08 & 24 & 39 & 42 & 45 & 55 & $\begin{array}{l}41,4- \\
42,67\end{array}$ \\
\hline Etiologia interpessoal & 17,34 & 5,57 & 7 & 14 & 17 & 21 & 38 & $\begin{array}{c}16,65- \\
18,04\end{array}$ & 30,62 & 4,76 & 15 & 28 & 31 & 34 & 42 & $\begin{array}{r}30,03- \\
31,21\end{array}$ \\
\hline
\end{tabular}

X = média; Escala total: 51-306 (Ponto médio 178); Autoritarismo: 11-66 (Ponto médio 38); Benevolência: 14-84 (Ponto Médio 7); Higiene Mental: 9-54 (Ponto Médio 31); Restrição Social: 10-60 (Ponto Médio 35); Etiologia Interpessoal: 7-42 (Ponto Médio 24) 
mais do que as mulheres na escala total ODM. Já no Brasil, os homens pontuam menos (0,246). Quanto maior for o tempo de formação para os enfermeiros portugueses o total da ODM diminui em 0,03 pontos. Já para os enfermeiros brasileiros, quanto maior for o tempo de formação, aumenta 2,3 pontos no total da ODM, sugerindo que os enfermeiros com maior tempo de formação estão menos propícios às atitudes negativas em ambos os países (Tabela 4).

Tabela 4. Correlação dos dados da ODM com variáveis sócio demográficas e profissionais, estudo Atitudes APS, PortoPortugal e São Paulo - Brasil $(n=500)$ enfermeiros

\begin{tabular}{|c|c|c|c|c|c|c|}
\hline \multirow{2}{*}{ Variáveis } & \multicolumn{3}{|c|}{ Portugal $(n=250)$} & \multicolumn{3}{|c|}{ Brasil $(n=250)$} \\
\hline & $p$-value & IC.io & IC.hi & $p$-value & IC.io & IC.hi \\
\hline Gênero (feminino) & $-0,085$ & $-3,201$ & 8,563 & 0,055 & $-6,58$ & 6,077 \\
\hline Estado civil & $-0,034$ & $-5,569$ & 5,608 & 0,023 & $-7,070$ & 1,897 \\
\hline Religião & 0,068 & $-4,241$ & 8,259 & $-0,067$ & $-10,374$ & 4,694 \\
\hline Habilitações acadêmicas & 0,003 & $-5,335$ & 4,955 & 0,006 & $-6,096$ & 10,833 \\
\hline Tempo de formação & 0,040 & $-1,955$ & 1,887 & $0,198^{\star \star}$ & $-0,207$ & 4,85 \\
\hline $\begin{array}{l}\text { Tempo de atuação } \\
\text { no atual serviço }\end{array}$ & 0,106 & $-0,531$ & 3,677 & 0,030 & $-3,642$ & 0,875 \\
\hline Carga horária & 0,052 & $-0,977$ & 0,435 & $0,324^{\star *}$ & 0,638 & 1,716 \\
\hline $\begin{array}{l}\text { Conhecimento } \\
\text { necessidades cuidado } \\
\text { em SM }\end{array}$ & $-0,103$ & $-5,871$ & 1,583 & 0,005 & $-1,348$ & 3,99 \\
\hline $\begin{array}{l}\text { Conhecimento papel dos } \\
\text { cuidados primários na } \\
\text { assistência em SM }\end{array}$ & $-0,102$ & $-4,701$ & 2,376 & $-0,056$ & $-2,536$ & 2,298 \\
\hline $\begin{array}{l}\text { Participação atividade } \\
\text { formativa no âmbito } \\
\text { da SM }\end{array}$ & $-0,0333$ & $-3,556$ & 6,505 & 0,076 & $-1,924$ & 7,87 \\
\hline $\begin{array}{l}\text { Experiência clínica com } \\
\text { pessoas com DM }\end{array}$ & $-0,078$ & $-10,988$ & 8,955 & 0,66 & $-1,433$ & 17,212 \\
\hline $\begin{array}{l}\text { Frequência que se } \\
\text { depara com pessoas } \\
\text { com DM }\end{array}$ & 0,034 & $-3,519$ & 1,112 & $0,277^{\star \star}$ & 2,795 & 10,004 \\
\hline $\begin{array}{l}\text { Avaliação necessidades } \\
\text { de saúde conduzida no } \\
\text { serviço }\end{array}$ & 0,054 & $-4,304$ & 2,571 & $-0,157^{\star \star}$ & $-3,401$ & 0,399 \\
\hline $\begin{array}{l}\text { Avaliação assistência } \\
\text { disponibilizada no } \\
\text { serviço }\end{array}$ & 0,010 & $-6,646$ & 2,117 & $-0,075$ & $-7,146$ & $-0,748$ \\
\hline
\end{tabular}

*A correlação no nível 0,001 (bilateral); **A correlação no nível 0,05(bilateral); ***Correlação significante para $p<0,05$; IC. $10=$ Intervalo de confiança inicial $I C . h i=$ Intervalo de confiança final

\section{Discussão}

A pesquisa foi realizada com enfermeiros que atuam em serviços heterogêneos quanto à realidade geográfica, demográfica, epidemiológica, financeira, número de atendimentos, recursos humanos, equipe de trabalho, processo de trabalho, produtividade e tipo de unidade, que asseguram cuidados de saúde de âmbito domiciliar, individual e familiar.
O contexto laboral dos enfermeiros, em ambos os países, demonstra diferenças relevantes quanto à carga horária de trabalho semanal, tempo de formação, tempo de atuação no atual serviço e as habilitaçóes acadêmicas que impactam no processo de cuidado de pessoas com doença mental. As semelhanças não fogem ao escopo da realidade da profissão enfermagem no contexto global, apresentando um perfil eminentemente do sexo feminino.

Os resultados encontrados expressam, de modo geral, opinióes positivas frente à pessoa com doença mental, similar a estudo desenvolvido com enfermeiros italianos, irlandeses, finlandeses, portugueses e africanos ${ }^{(11,12)}$ exceto, na dimensão Autoritarismo, similar a realidade de enfermeiros lituanos e chineses, ${ }^{(6,12)}$ conduzidos com outra escala de avaliação, realidade também encontrada no Brasil, com uso da escala ODM. ${ }^{(14,18)}$ Todavia, apesar da atitude dos enfermeiros brasileiros ser negativa na dimensão Autoritarismo, isto não é suficiente para indicar a visão de que pessoas com doença mental são inferiores e requerem uma abordagem impositiva por parte desses profissionais.

Os enfermeiros brasileiros detêm menor tempo de formação, logo, são ligeiramente mais autoritários que os portugueses. Estudos apontaram que a evolução formativa superior a doze anos possibilita atitudes menos negativas na dimensão Autoritarismo $^{(19)}$ e que enfermeiros mais instruídos são mais conscientes dos estigmas que envolvem as pessoas com doenças mentais em relação aos com menor instrução, o que resulta em atitudes menos discriminantes. ${ }^{(4)}$ Ademais, quanto mais elevados forem os níveis de ensino que o profissional tem, maior é o impacto positivo frente à redução dos estigmas e rótulos. ${ }^{(20)}$ Portanto, projetar currículos destinados a restringir crenças e atitudes negativas em relação à doença mental na formação dos enfermeiros pode minimizar fatores que influenciam esses fenômenos. ${ }^{(2)}$

Detendo atitude de autoritarismo, os enfermeiros brasileiros acreditam que pessoas com doença mental são incapazes de tomar decisóes sobre suas próprias vidas ${ }^{(21,22)}$ o que representa uma adversidade no campo da saúde/saúde mental, que conduz a estigmatização, discriminação, obstáculos na recuperação, na inserção 
social, ${ }^{(8)}$ e reproduz a lógica manicomial, amplamente confrontada pelo processo de reforma do modelo assistencial em saúde mental no dois países. ${ }^{(23,24)}$

Comparando as duas realidades, os enfermeiros brasileiros também apresentam atitudes mais negativas nas dimensões Restrição Social e Etiologia Interpessoal, que designam que a pessoa com doença mental deve ser restrita do contexto social, ${ }^{(25)} \mathrm{e}$ que o adoecimento mental ocorre em função de escolhas individuais conduzidas ao longo da vida, tendo como pano de fundo as relaçóes interpessoais problemáticas, ${ }^{(26)}$ dados que encontram respostas devido ao menor tempo de formação profissional. (19) Salienta-se que, quando o usuário recebe atitudes negativas, as possibilidades futuras para o enfermeiro reverter estas para uma atitude positiva diminuem e impactam em seu tratamento.

A frequência que os enfermeiros se deparam com pessoas com doença mental é elevada na realidade brasileira, todavia não possibilitou atitudes mais positivas, predominando posiçóes autoritárias, restritivas e discriminatórias. Possivelmente, esses achados devem-se, também, ao seu menor tempo de formação, comparado aos portugueses. Contudo, os brasileiros são críticos ao reconhecerem a importância do seu papel profissional nos cuidados primários em saúde mental, a exemplo dos portugueses. Ao estudar os efeitos de estratégias diretas e indiretas das atitudes estigmatizantes na constituição de um programa de saúde mental sem estigmas, autores ${ }^{(25)}$ detectaram que o tempo de formação profissional amplia a desconstrução de crenças discriminatórias, a compreensão das individualidades e atenção abrangente das necessidades da pessoa com doença mental.

Pesquisa portuguesa afirma que o aumento do aporte de experiência clínica em saúde mental é passível de produzir atitudes mais positivas, em relação às pessoas com doença mental. ${ }^{(27)}$ No quesito experiência clínica com pessoas com doenças mentais, não houve correlação em relação aos dois países, ainda assim, os enfermeiros brasileiros afirmam que seus conhecimentos são muito adequados quanto às necessidades de cuidados de saúde dessas pessoas, e a avaliação da assistência disponibilizada pelos seus serviços, bem superior à que os enfermeiros portugueses consideram.
A dimensão Benevolência apresentou-se acima da média para ambas as nacionalidades, ainda que ligeiramente superior na realidade portuguesa, e está diretamente relacionada com o reconhecimento que os enfermeiros têm das necessidades de cuidados de pessoas com doenças mentais dentro de suas circunstâncias de trabalho, dado corroborado em estudo brasileiro. ${ }^{(19)}$

Ter atitudes benevolentes significa exercer uma visão paternalista e protecionista frente à pessoa com doença mental, entretanto, questiona-se se essa atitude potencializa ou não uma assistência mais qualificada e confere poder contratual a pessoa com doença mental. Destaca-se, contudo, que nas enfermeiras predominam esse perfil, reflexo da singularidade paternalista e gentil em relaçáo à doença mental, cujas origens derivam da religião e do humanismo, e não da ciência. ${ }^{(28)}$

O processo de trabalho dos enfermeiros brasileiros implica em uma carga horária semanal superior a dos portugueses e associa-se a atitudes mais negativas. A exposição da equipe de enfermagem a longas jornadas de trabalho compromete a qualidade do cuidado, ${ }^{(29)}$ expóe o profissional a rotinas mais exaustivas, conduzindo o enfermeiro a priorizar metas e resultados. Os desgastes nesse percurso podem interferir na relação que trava com o usuário do serviço, no estreitamento de vínculos, no raciocínio clínico a acerca das necessidades de saúde, diminuindo sua atitude benevolente.

$\mathrm{Na}$ realidade dos enfermeiros brasileiros as atitudes negativas provêm das opinióes negativas, e são geradas pelo menor tempo de atuação no serviço. Como os serviços, campos de pesquisa utilizados, são de gestão indireta, ou seja, administrados por Organizaçóes Sociais de Saúde, tem-se que a rotatividade é elevada e não há estabilidade, pois os contratos de trabalho são conduzidos de acordo com as leis trabalhistas do país. ${ }^{(30)}$

O redirecionamento da Política de Saúde Mental Brasileira preconiza uma rede de serviços integrada e ampliada, orientada pelas diretrizes da Rede de Atenção Psicossocial, ${ }^{(31)}$ que tem nos componentes de serviços primários um dos eixos para sua efetivação, e clama aos enfermeiros desse campo de prática que, diariamente lidam com pessoas com doenças 
mentais, um olhar mais qualificado. Porém, as atitudes positivas destes ainda não avançaram o suficiente, frente a outras realidades, uma vez que são mais negativas do que os enfermeiros portugueses. Mesmo assim, os enfermeiros brasileiros consideram que conduzem avaliação das necessidades de saúde das pessoas com doenças mentais adequadamente, entendimento que pode comprometer suas condutas terapêuticas.

O campo dos cuidados primários não conta com enfermeiros especialistas em saúde mental, e nem deve, posto que o enfermeiro generalista tem por compromisso de formação assistir o indivíduo em sua integralidade. Entretanto, em muitas graduaçóes, conteúdos dessa esfera não são abordados, e, quando o são, mostram-se fracos e restritos a lógica hospitalocêntrica/biomédica ${ }^{(32)}$ e pouco interesse desperta nos futuros profissionais. Diante dessa conjuntura, a educação permanente é uma condição sine qua non para o enfermeiro identificar e intervir precocemente junto a pessoa em sofrimento psíquico, e/ou referenciá-la aos serviços especializados. ${ }^{(33,34)}$

Estudo aponta que há uma conexão entre uma visão menos crítica frente o cuidado à pessoa com doença mental, a inexperiência no campo atual de trabalho, e/ou a ausência de treinamento adicional em saúde mental, durante ou após a formação de enfermeiros mais jovens, que resultam em atitudes mais estigmatizantes e até mesmo em medo no lidar com essa clientela. ${ }^{(10)}$

Vivências práticas produzem mudanças mais positivas do que as teóricas em relação a pessoa com doença mental. ${ }^{(33)}$ Por outro lado, o contato com pessoas com doenças mentais tem impacto positivo nas crenças e atitudes, especificamente ao nível das atitudes estigmatizantes e comportamentos discriminatórios. ${ }^{(5)}$ Considera-se que, os enfermeiros desempenham um papel fundamental na luta contra o estigma e a discriminação, para tal, torna-se necessário mais investimento na capacitação profissional dos enfermeiros de cuidados primários para fortalecer suas atitudes positivas. ${ }^{(10)}$

Posto que, as necessidades de cuidados em saúde mental perpassam todos os níveis de atenção à saúde e devem ser respeitadas, em ambos os países investigados, os enfermeiros apresentam experiên- cia de trabalho com pessoas com doenças mentais. Sendo maior em Portugal, possivelmente, por terem um tempo de atuação no serviço atual muito superior (10 a 20 anos) do que os brasileiros (1 a 5 anos.

Essa realidade configura-se, atualmente, com elevada demanda na Atenção Primária à Saúde, campo de cuidado que solicita por possibilidades de assistência psicossocial responsiva e resolutiva, coerente com a lógica da integralidade e longitudinalidade dos sistemas de saúde e das políticas públicas vigentes no mundo. A integração dos cuidados de saúde mental aos primários melhora o acesso dos usuários aos serviços de saúde como um todo, minimiza estigmas, discriminação, promove o respeito pelos direitos humanos e representa um importante indicador da qualidade assistencial.

Apesar da limitação do estudo recair no fato da coleta de dados ter sido conduzida por meio de questionários eletrônicos, que dificulta o reconhecimento da população total, é importante destacar que este estudo é pioneiro na investigação das atitudes de enfermeiros de cuidados primários do Brasil e de Portugal frente à pessoa com doença mental, e tem a força para direcionar a assistência de enfermagem em saúde mental. Ademais, subsidia programas de capacitação que qualifiquem equipes, com vistas a desconstruir o paradigma excludente e fortalecer o modelo psicossocial de cuidado.

\section{Conclusão}

Este estudo revelou que de modo geral, as atitudes entre os enfermeiros de cuidados primários nos dois países em relação às pessoas com doença mental são positivas, exceto nas dimensóes Autoritarismo, maior no Brasil. Em Portugal, as dimensóes Benevolência e Ideologia de Higiene Mental predominam. As experiências de cuidados apresentaram diferenças importantes nas atitudes entre os enfermeiros portugueses e brasileiros. Variáveis como menor carga horária semanal, maior tempo de formação, de atuação no serviço e frequência de experiência clínica em saúde mental, influenciam de maneira positiva nas atitudes. A opinião que os enfermeiros têm sobre seu papel na avaliação da assistência e das necessidades de cuidados de saúde a 
pessoa com doença mental também tem impacto positivo ou negativo nas atitudes laborais destes. As evidências encontradas entre as duas realidades são distintas, porém semelhantes quanto ao processo histórico, portanto, possibilitam reconfigurar saberes e práticas de cuidados, subsidiar uma práxis isenta de concepçóes estereotipadas e avançar na construçáo de um novo paradigma no campo dos cuidados primários.

\section{Colaborações}

\section{Nóbrega MPSS e Fernandes CSNN participaram da} concepção do projeto, análise e interpretação dos dados, revisão crítica relevante do conteúdo intelectual e aprovação final da versão a ser publicada. Duarte E, Chaves SCS participaram da análise, interpretação dos dados, redação do artigo e aprovação final da versão a ser publicada. Moreira WC participou da análise, interpretação dos dados, redação do artigo, revisão crítica relevante do conteúdo intelectual e aprovação final da versão a ser publicada.

\section{Referências}

1. Hunter KN, Rice S, MacDonald J, Madrid J. What are the best predictors of opinions of mental illness in the Indian population? Int J Ment Health. 2014;43(2):35-51.

2. Tambag $H$. Effects of a psychiatric nursing course on beliefs and attitudes about mental illness. Int J Caring Sci. 2018;11(1):420-6.

3. Staniulienè V, Chambers M, Kantaris X, Kontio R, Kuosmanen L, Scott A, et al. The feelings and thoughts of mental health nurses concerning the management of distressed and disturbed in-patients: A comparative qualitative European study. Open J Nurs. 2013;3(6):426-36.

4. Neupane D, Dhakal S, Thapa S, Bhandari PM, Mishra SR. Caregivers' Attitude towards People with Mental IIIness and Perceived Stigma: A Cross-Sectional Study in a Tertiary Hospital in Nepal. PLoS One. 2016;11(6):e0158113.

5. Gil I, Santos, J, Loureiro L. Estigma em estudantes de enfermagem: antes e depois do contacto com pessoas com transtornos mentais. Rev Enferm UERJ. 2016; 24(1):e12309.

6. Ma Z, Huang H, Nie G, Silenzio VM, Wei B. Attitude towards Mental IIIness among Primary Healthcare Providers: A Community-Based Study in Rural China. BioMed Res Int. 2018;2018:8715272.

7. Hsiao CY, Lu HL, Tsai YF. Factors influencing mental health nurses' attitudes towards people with mental illness. Int J Ment Health Nurs. 2015;24(3):272-80.

8. Mårtensson G, Jacobsson JW, Engström M. Mental health nursing staff's attitudes towards mental illness: an analysis of related factors. J Psychiatr Ment Health Nurs. 2014;21(9):782-8.
9. Henderson C, Noblett J, Parke H, et al. Mental health-related stigma in health care and mental health-care settings. Lancet Psychiatry. 2014;1(6):467-82.

10. Ihalainen-Tamlander $N$, Vähäniemi $A$, Löyttyniemi $E$, Suominen $T$, Välimäki M. Stigmatizing attitudes in nurses towards people with mental illness: a cross-sectional study in primary settings in Finland. J Psychiatr Ment Health Nurs. 2016;23(6-7):427-37.

11. Dube FN, Uys LR. Integrating mental health care services in primary health care clinics: a survey of primary health care nurses' knowledge, attitudes and beliefs. S Afr Fam Pract. 2016;58(3):11925.

12. Chambers $M$, Guise V, Välimäki $M$, Botelho MA, Scott A, Staniuliené V, et al. Nurses' attitudes to mental illness: a comparison of a sample of nurses from five European countries. Int J Nurs Stud. 2010;47(3):35062.

13. Pedrão LJ, Tavares RR. Análise comparativa da opinião de enfermeiros de diferentes unidades psiquiátricas sobre a pessoa em sofrimento psíquico. Cogitare Enferm. 2003;8(1):75-86.

14. Soares MH, Ruzzon ED, Bortoletto MS. Concepção de profissionais de saúde que atuam em emergência de saúde mental. SMAD Rev Eletrônica Saúde Mental Álcool Drog. 2014;10(2):85-92.

15. Nóbrega MD, Silva GB, Sena AC. Psychosocial rehabilitation in the west network of the municipality of São Paulo: potentialities and challenges. Rev Gaucha Enferm. 2018;39:e20170231.

16. Struening EL, Cohen J. Factorial invariance and other psychometric characteristics of five opinions about mental illness factors. Educ Psychol Meas. 1963;23(2):289-98.

17. Rodrigues CR. Atitudes frente à doença mental: estudo transversal de uma amostra de profissionais de saúde. 1983. 148 p. [tese]. Ribeirão Preto: Faculdade de Medicina de Ribeirão Preto; 1983.

18. Gonçalves AM, Vilela SC, Terra FS. Atitudes de profissionais da Rede de Atenção Psicossocial frente ao adoecimento mental. Rev Rene. 2017;18(5):647-54

19. Abramenko L, Lovisi GM, Fonseca DL, Abelha L. Atitudes dos trabalhadores de saúde mental em relação aos pacientes psiquiátricos em uma cidade do interior do Estado do Rio de Janeiro. Cad Saude Colet. 2017;25(2):169-76.

20. Bilge A, Çam 0. The Fight against Stigma toward Mental IIIness. Derleme/Rewiev Article. Turk Silahli Kuvvetleri Koruyucu Hekim Bul. 2010;9(1):71-8. Turkish.

21. Santos SS, Soares MH, Hirata AG. Attitudes, knowledge, and opinions regarding mental health among undergraduate nursing students. Rev Esc Enferm USP. 2013;47(5):1195-202.

22. Ahmead MK, Rahhal AA, Baker JA. The attitudes of mental health professionals towards patients with mental illness in an inpatient setting in Palestine. Int J Ment Health Nurs. 2010;19(5):356-62.

23. Passos RG, Portugal $S$. Breve balanço da política de saúde mental: análise comparativa Brasil e Portugal a partir da experiência das residências terapêuticas. Rev Pol Públ. 2015;19(1):91-102.

24. Brasil. Ministério da Saúde. Lei n. ${ }^{0} 10.216$ de 6 de abril de 2001. Dispõe sobre a proteção de transtornos mentais e redireciona o modelo assistencial em saúde mental [Internet]. Brasília (DF): Ministério da Saúde; 2001. [citado 2020 Mar 15]. Disponível em: http://www. planalto.gov.br/ccivil_03/LEIS/LEIS_2001/L10216.htm

25. Oliveira S, Carolino L, Paiva A. Programa saúde mental sem estigma: efeitos de estratégias diretas e indiretas nas atitudes estigmatizantes. Rev Port Enferm Saude Mental. 2012;(8):30-57. 
26. Yildirim M, Demirbuken I, Balci B, Yurdalan U. Beliefs towards mental illness in Turkish physiotherapy students. Physiother Theory Pract. 2015;31(7):461-5.

27. Martinho J, Pires R, Carvalho JC, Pimenta G. Formação e desenvolvimento de competências de estudantes de enfermagem em contexto de ensino clínico em saúde mental e psiquiatria. Rev Port Enferm Saúde Mental. 2014 (Ed Esp 1): 97-102.

28. Gur K, Kucuk L. Females' attitudes toward mental illness: a sample from rural istanbul, turkey. Iran Red Crescent Med J. 2016;18(5):e22267.

29. Kunaviktikul W, Wichaikhum 0 , Nantsupawat A, Nantsupawat $R$, Chontawan R, Klunklin A, et al. Nurses' extended work hours: Patient, nurse and organizational outcomes. Int Nurs Rev. 2015 ;62(3):386-93.

30. Pilotto BS, Junqueira V. Organizações Sociais do setor de saúde no estado de São Paulo: avanços e limites do controle externo. Serv Soc Soc. 2017;(130):547-63.
31. Brasil, Ministério da Saúde. Portaria n. ${ }^{\circ} 3.088 / G M$ de 23 de dezembro de 2011. Institui a Rede de Atenção Psicossocial para pessoas com sofrimento ou transtorno mental e com necessidades decorrentes do uso de crack, álcool e outras drogas, no âmbito do Sistema Único de Saúde. Brasília (DF): Ministério da Saúde; 2011. [citado 2020 Mar 15]. Disponivel em: http://bvsms.saude.gov.br/bvs/saudelegis/gm/2011/ prt3088_23_12_2011_rep.html

32. Carneiro LA, Porto CC. Saúde mental nos cursos de graduação: interfaces com as diretrizes curriculares nacionais e com a reforma psiquiátrica. Cad Bras Saúde Mental. 2014;6(14):150-67.

33. Yuksel EG, Taskin EO. The attitudes and knowledge of the physicians and the medical school students towards mental disorders in Turkey. Anadolu Psikiyatri Derg. 2005;6(2):113-21.

34. Fernandes CS, Santos WB, Moreira WC, Vargas D, Nóbrega MD. Opinions on mental illness from the perspective of primary care nurses in Portugal. Rev Gaúcha Enferm. 2019;40:e20190034. 